\title{
Oto-onycho-peroneal syndrome: confirmation of a syndrome
}

\author{
Koenraad Devriendt, Daniel Stoffelen, Rudolf Pfeiffer, Anita Leys, Jean-Pierre Fryns
}

Centre for Human
Genetics, University
Hospital Leuven,
Herestraat 49, B-3000
Leuven, Belgium
K Devriendt
J-P Fryns
Department of
Traumatology,
University Hospital
Leuven, Herestraat 49,
B-3000 Leuven,
Belgium
D Stoffelen

Department of Ophthalmology, University Hospital Leuven, Herestraat 49, B-3000 Leuven,

Belgium

A Leys

\section{Institut für \\ Humangenetik und Anthropologie der Universität \\ Erlangen-Nürnberg, Germany \\ R Pfeiffer}

Correspondence to: Dr Devriendt.

Received 12 September 1997 Revised version accepted for publication

28 November 1997

\begin{abstract}
We report two sibs with a similar syndrome of abnormal external ears, peculiar facial features, nail hypoplasia, a bilateral fibrous fusion of the outer third of the clavicle and the scapular spine, and the absence of a normal acromioclavicular joint. The present patients represent the fourth and fifth cases of the oto-onychoperoneal syndrome (MIM 259780).

(f Med Genet 1998;35:508-509)
\end{abstract}

Keywords: nail hypoplasia; shoulder malformation; external ear; autosomal recessive

\section{Case reports}

The index patient of this family is a 41 year old female. At the age of 40 years, she was referred for progressive shoulder dysfunction, pain on movement, and limited motion. On inspection, the acromion appeared abnormal. A skin dimple was present on the dorsolateral side of the shoulder bilaterally. Radiographically, a bilateral, wide, bony, and fibrous fusion between the distal third of the clavicle and the spine of the scapula was observed, with absence of a normal acromioclavicular joint (fig 1). Examination under anaesthesia showed hyperlaxity of the shoulder and, on arthroscopy, dysplasia of the glenoid was detected. On further clinical examination, peculiar facial features were noted, with a prominent nose and a columella extending below the nasal alae (fig $2 \mathrm{~A}$ ). The palpebral fissures were relatively narrow. The ears were normally set, but large, with an unfolded helix and a prominent anthelix at the lower end (fig 2A). There was bilateral nail hypoplasia of the index fingers (fig 3 ). The nails of the first and second toes were dysplastic. She had grey hair from the age of 25 years, but no

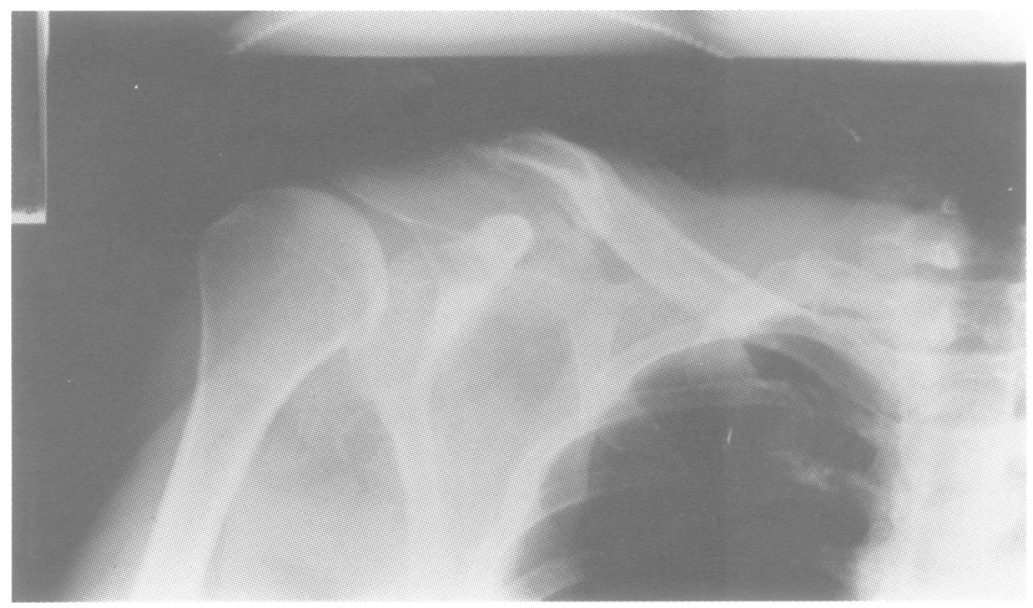

Figure 1 Radiograph of the left shoulder of patient 1 (front view). Note the wide, bony, and fibrous fusion between the distal third of the clavicle and the spine of the scapula with absence of a normal acromioclavicular joint. premature hair loss. The teeth were absent from the age of 23 years owing to caries. Clinically, there were no abnormalities of the other joints. Radiographs of the elbows, knees, and ankles were normal. Radiographs of the hands showed short terminal phalanges of the index fingers with prominent tufting. Radiographs of the feet showed hypoplasia of the middle phalanges of several toes and an abnormal shape of the first phalanx of the first toes, with a V shaped distal end. Biometry and intelligence were normal. Eye examination showed full vision with correction of mild astigmatism. The anterior segments appeared normal, with relatively flat anterior chambers and pupillary membrane residues in each eye. The horizontal diameter of the cornea was $11 \mathrm{~mm}$ (normal value above $10 \mathrm{~mm}$ ). Ultrasonographic measurement of axial length showed microphthalmia: right eye $20.1 \mathrm{~mm}$ and left eye 19.9 $\mathrm{mm}$ ( $>2$ SD below normal). The posterior segments of the eyes were normal. Karyotype on white blood cells was normal $46, \mathrm{XX}$ after $\mathrm{G}$ and $\mathrm{T}$ banding.

The second patient is a younger sister of patient 1 , aged 23. She has similar clinical features to her sister, with the same facial features and the same abnormal shape of the ears (fig 2B). She complained of minor shoulder pain without diminished mobility. Radiographs showed a similar malformation of the joint between the clavicle and scapula as seen in her sister. The nails of the index fingers and second toes were hypoplastic and the nails of several other fingers were equally poorly formed. Her hair was normal. She had several deciduous teeth. Biometry and intelligence were normal. Eye examination showed the same minor anomalies as seen in her sister, including relatively flat anterior chambers, bilateral pupillary membrane residues, relatively small corneas (horizontal diameter of $11 \mathrm{~mm}$ ), and short eyes (axial length $20.5 \mathrm{~mm}$ in the right eye, $20.4 \mathrm{~mm}$ in the left eye). The eye fundus showed an unusual pattern of pigmentation with patchy depigmentation and enhanced visualisation of the choroid, which was most pronounced in the inferior section of the right eye.

These two patients are from a sibship of 10 children. Two boys died during infancy, one from prematurity, the other from an unknown cause. They did not have nail or ear abnormalities. No other family members have ear, nail, or skeletal abnormalities. Patient 1 has three healthy, unaffected children. The parents are not known to be consanguineous, but originate from the same small village. 

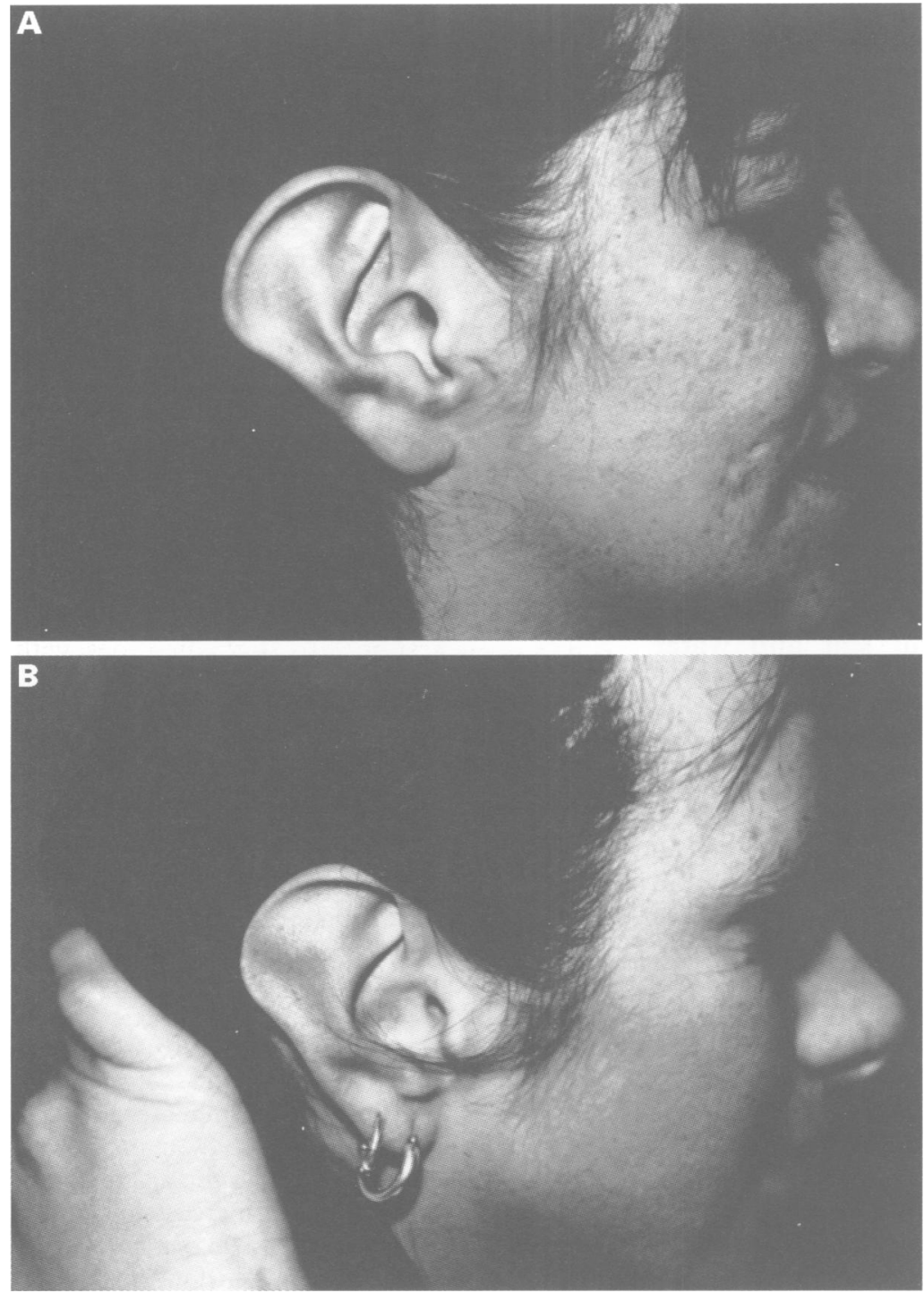

Figure 2 (A) Profile of patient 1. (B) Profile of patient 2. Note the similar features in both sibs, with abnormal shape of the ears and nose, with the columella extending below the nasal alae. (Photographs reproduced with permission.)

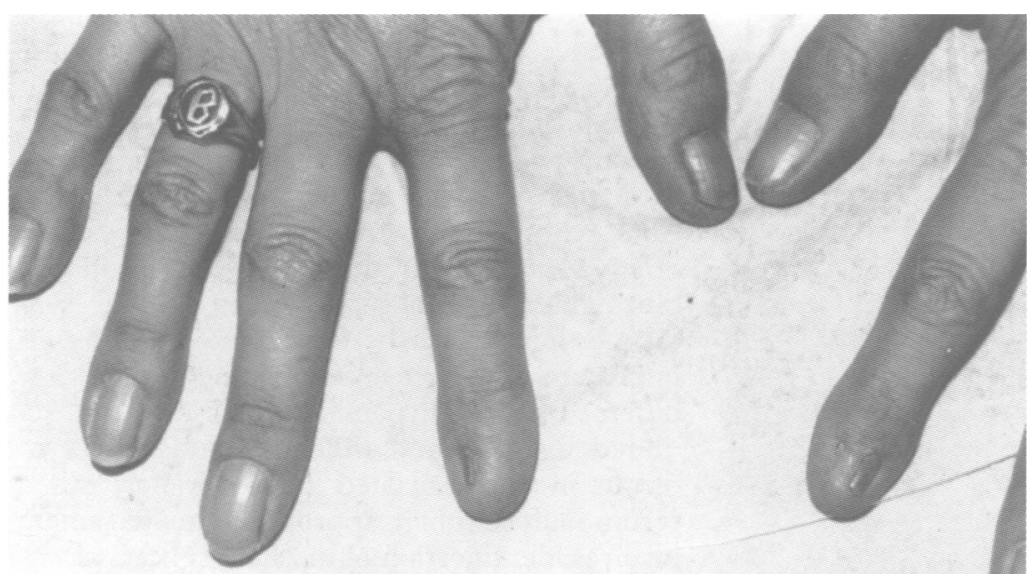

Figure 3 Nail hypoplasia of the index fingers in patient 1.

Discussion

We report two female sibs with the same clinical features including congenital nail hypoplasia, dysmorphic facial features with distinct ear anomalies, and a unique malformation of the shoulder joint.
This constellation of malformations bears striking resemblance to the abnormalities found in a male patient reported by Leiba $e t a l^{1}$ and two sibs described by Pfeiffer. ${ }^{2}$ All patients share similar facial features, especially the malformed ears. Nail hypoplasia involved several fingers and toes in the patients reported by Pfeiffer, ${ }^{2}$ whereas it was limited to the first or second toes and fingers in the present patients and the patient of Leiba et al. ${ }^{1}$

The skeletal abnormalities in the five patients described so far are diverse. In the present patients, a shoulder malformation was the major manifestation, but was also described in the other patients; the clavicles were noted to be abnormally straight in the patients of Pfeiffer, ${ }^{2}$ and the patient reported by Leiba et $a l^{1}$ also had a malformation of the lateral part of the clavicle and of the acromion. In the patients reported by Pfeiffer, ${ }^{2}$ the clinical picture was dominated by severe joint deformities, leading to popliteal and inguinal pterygia and club feet. Hypoplasia of the proximal fibula was present in the older sib, whereas in the younger patient the fibula was absent, leading to the designation "peroneal" of the oto-onycho-peroneal syndrome. The younger child has died but the cause of death is unknown to us. The older sib underwent several orthopaedic interventions and he finally succeeded in walking. His intelligence is normal.

The patient reported by Leiba et al had nystagmus and eye malformations, including bilateral coloboma of the retina, left microcornea, and pupillary membrane residues. Interestingly, at the age of 8 years, eye examination of the oldest patient reported by Pfeiffer $^{2}$ showed a hyperopic right eye with grey-green macula alterations. The left eye was amblyopic and showed similar macular alterations and a retinochoroidal coloboma. A detailed eye examination in the present patients showed only minor changes, not associated with any clinical problems.

The oldest patient reported by Pfeiffer $^{2}$ had bilateral mixed hearing loss (with predominant perceptive hearing loss) of $60 \mathrm{~dB}$; the younger patient could not be examined. Hearing was normal in the other patients.

This condition represents a clinically identifiable entity, characterised by a specific external ear malformation, nail hypoplasia, and variable skeletal malformations. It may be associated with deafness and eye manifestations. The disorder is most likely autosomal recessive, given the occurrence in sibs of both sexes with normal parents, and in the case reported by Leiba et al ${ }^{1}$ with consanguineous parents.

1 Leiba S, Grünebaum M, Savir H, Ber A. Oculootonasa malformations associated with osteoonychodysplasia. Birth Defects 1975;11:67-73.

2 Pfeiffer R. The oto-onycho-peroneal syndrome. A probably new genetic entity. Eur f Pediatr 1982;138:317-20. 